\title{
Photon-Assisted Transport Through a Double Quantum Dot With a Time-dependent Interdot Barrier
}

\author{
Ph. BruneГC. BruderГand H. Schoeller \\ Institut für Theoretische Festkörperphysik, Universität Karlsruhe, D-76128 Karlsruhe, Germany
}

\begin{abstract}
We study transport through two ultrasmall quantum dots with discrete energy levels to which a time-dependent field is applied (e.g.Tmicrowaves). The AC field causes photon-assisted tunneling and also transitions between discrete energy levels of the dot. Using the Floquet-matrix technique we propose an electron pump consisting of two serially coupled single-level quantum dots with a time-dependent interdot barrier. We compare our results to recent experiments by Fujisawa and Tarucha.
\end{abstract}

Many aspects of the Coulomb blockade are now well understood. Recently has come up Tviz. Ttime-dependent transport through small quantum dots. High-frequency $\mathrm{AC}$ voltages can be applied to mesoscopic structures (e.g. Tin the form of microwaves). They lead to photon-assisted tunneling $\Gamma$ i.e. Telectrons can overcome the Coulomb blockade by absorbing photons from the external field. This has become a very active area recently both experimentally ${ }^{1-6}$ and theoretically ${ }^{7-13}$.

In this work Twe will study transport through two ultrasmall quantum dots with discrete energy levels to which a time-dependent field is applied. The electron interaction in the dots is taken into account by the Coulomb blockade model. The dots are weakly coupled to source and drain reservoirs by tunnel junctions. Time-dependent gate voltages lead to photon-assisted tunneling. We propose an electron pump consisting of two serially coupled single-level quantum dots strongly coupled by time-dependent fields. Transport through a double dot with a time-dependent interdot barrier has been measured recently by Fujisawa 
and Tarucha ${ }^{5}$.

Our system consists of two interacting quantum dots in a time-dependent periodic field coupled to two reservoirs by tunnel junctions $\Gamma$ see Fig. 1 . We will use the time-dependent Hamiltonian $H(t)=H_{r e s}+H_{d o t}(t)+H_{t u n}{ }^{9}$. Here $H_{r e s}(t)=\sum_{k, \alpha, \sigma} \epsilon_{k \alpha \sigma} c_{k \alpha \sigma}^{\dagger} c_{k \alpha \sigma}$ describes noninteracting electrons in the reservoirs $\{\alpha\}=\{L, R\} \Gamma c_{k \alpha \sigma}^{\dagger} / c_{k \alpha \sigma}$ are the creation/annihilation operators of an electron with momentum $k$ and spin $\sigma$ in the reservoir $\alpha$. The dot Hamiltonian is given by

$$
H_{d o t}(t)=\sum_{\alpha, \sigma} \epsilon_{\alpha \sigma}(t) d_{\alpha \sigma}^{\dagger} d_{\alpha \sigma}+\sum_{\sigma} w(t) d_{L \sigma}^{\dagger} d_{R \sigma}+h . c .+H_{c h}\left(N_{d o t}\right)
$$

where $d_{\alpha \sigma}^{\dagger} / d_{\alpha \sigma}$ create/annihilate electrons with spin $\sigma$ in $\operatorname{dot} \alpha$. The energy of the level in dot $\alpha$ is given by $\epsilon_{\alpha \sigma}(t)=\epsilon_{\alpha \sigma}^{0}+\Delta_{D} \cos (\omega t)$ Twhere the time dependence is taken into account by a periodic shift of the level. The coupling strength of the field to the dot is given by $\Delta_{D}$. The time-dependent transition matrix element $w(t)=\Delta_{0}+\Delta \cos (\omega t)$ describes transitions between the dots i.e. Ttransitions that do not change the total number of electrons in the dots. The Coulomb interaction between electrons in the dot is taken into account by the Coulomb-blockade model $H_{c h}\left(N_{d o t}\right)=E_{C} N_{d o t}^{2}$. Here $\Gamma N_{d o t}=N_{L}+N_{R}=\sum_{\alpha=L / R, \sigma} d_{\alpha \sigma}^{\dagger} d_{\alpha \sigma}$ is the particle number in the $\operatorname{dots} \Gamma E_{C}=2 e^{2} / 3 C$ is the charging energy with $C=C_{L}+C_{R}+2 C_{g}$. The capacitance $C_{M}$ between the dots is chosen to be $C_{M}=C / 2$ and we set $C_{L}=C_{R}$. Note that we have included the time-dependent parts $\left[2 E_{C} n_{0}^{L / R}(t)+E_{C} n_{0}^{R / L}(t)\right] N_{L / R}$ of the original $H_{c h}^{\text {orig }}\left(N_{d o t}, t\right)=E_{C} N_{d o t}^{2}+\left[2 E_{C} n_{0}^{L}(t)+E_{C} n_{0}^{R}(t)\right] N_{L}+\left[2 E_{C} n_{0}^{R}(t)+E_{C} n_{0}^{L}(t)\right] N_{R}$ in the energies $\epsilon_{\alpha \sigma}(t)$. The $e n_{0}^{\alpha}(t)=C_{\alpha} V_{\alpha}(t)+C_{g} V_{g \alpha}(t)$ are related to the polarization charges produced by the voltages of the left and right reservoirs $\mu_{L / R}$ as well as the time-dependent gate voltages $e V_{g \alpha}(t)=V_{g \alpha}+\Delta_{g \alpha} \cos (\omega t)$ applied to the left and right dot by the capacitance $C_{g}$. The tunneling part is given by $H_{t u n}=\sum_{k, \alpha, \sigma} T_{k \alpha} c_{k \alpha \sigma}^{\dagger} d_{\alpha \sigma}+$ h.c. .where $T_{k \alpha}$ denotes the tunneling matrix element.

We now generalize the work of Stafford and Wingreen ${ }^{11}$ to the case where the height of the tunneling barrier also depends on time. The time-dependent Schrödinger equation is solved by the Floquet-matrix approach ${ }^{14}$. It is now possible to transform the tunneling Hamiltonian 
and calculate the current by the master equation technique ${ }^{9,13}$. The resulting dependence of the pumped current on the frequency and the amplitude of the applied microwaves is plotted in Fig. 2. Here and in the rest of the paper we used $E_{C}=75 \Gamma \Gamma \Gamma_{L}=\Gamma_{R}=\Gamma$ is the tunneling rate. In Fig. 2 we set $\epsilon_{L}^{0}=-10 \Gamma \Gamma \epsilon_{R}^{0}=10 \Gamma \Gamma \mu_{L}=\mu_{R}=0 \Gamma T=5 \Gamma$.

In Fig. 3 we show a comparison of our results with a recent experiment ${ }^{5}$.

In conclusion Twe have calculated the photon-assisted transport through a double quantum dot with a time-dependent interdot barrier. We have proposed a new electron pump and found qualitative agreement with recent experimental results by Fujisawa and Tarucha. 


\section{REFERENCES}

${ }^{1}$ L. P. Kouwenhoven et al.ГPhys. Rev. B 50Г2019 (1994).

${ }^{2}$ L. P. Kouwenhoven et al.ГPhys. Rev. Lett. 73 Г3443 (1994).

${ }^{3}$ R. H. Blick et al.ГAppl. Phys. Lett. 67Г3924 (1995); R. H. Blick et al.ГSurf. Science 361 595 (1996).

${ }^{4}$ K. Fujii et al.ГPhysica B 227Г98 (1996).

${ }^{5}$ T. Fujisawa and S. TaruchaГSuperlattices and Microstructures 21Г247 (1996); Jpn. J. Appl. Phys. 36Гxxxx (1997).

${ }^{6}$ T. H. Oosterkamp et al. ГPhys. Rev. Lett. 78Г1536 (1997).

${ }^{7}$ M. BüttikerГA. PrêtreГand H. ThomasГPhys. Rev. Lett. $70 \Gamma 4114$ (1993).

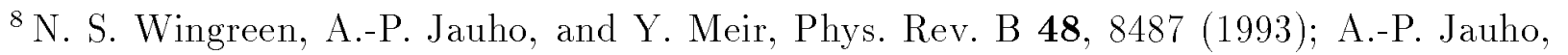
N. S. WingreenTand Y. MeirTPhys. Rev. B 50Г5528 (1994).

${ }^{9}$ C. Bruder and H. SchoellerГPhys. Rev. Lett. 72Г1076 (1994); C. Bruder and H. Schoeller $\Gamma$

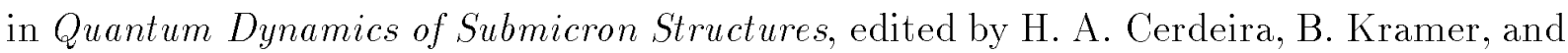
G. SchönГNATO ASI Ser. EГVol. 291 (KluwerГDordrechtГ1995)Гp. 383.

10 T. H. Stoof and Y. V. Nazarov TPhys. Rev. B 53Г1050 (1996).

${ }^{11}$ C. A. Stafford and N. S. Wingreen $\Gamma$ Phys. Rev. Lett. 76Г1916 (1996).

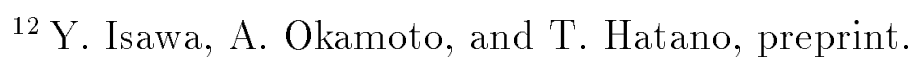

${ }^{13}$ Ph. BruneГC. BruderTand H. SchoellerГPhys. Rev. B (Aug. 15Г1997).

${ }^{14}$ J. H. ShirleyГPhys. Rev. 138ГВ979 (1965). 


\section{FIGURES}

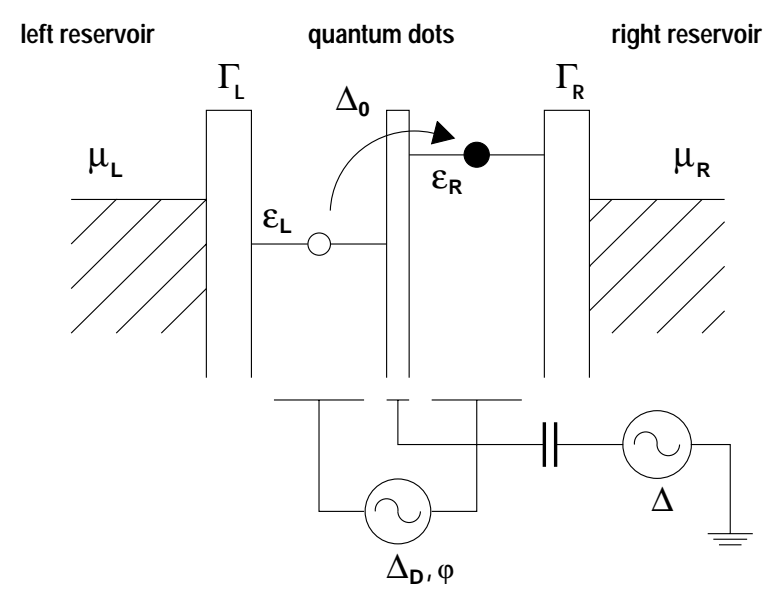

FIG. 1. Energy landscape of two serially coupled quantum dots connected by a weak time-dependent barrier (periodicity $\omega$ ).

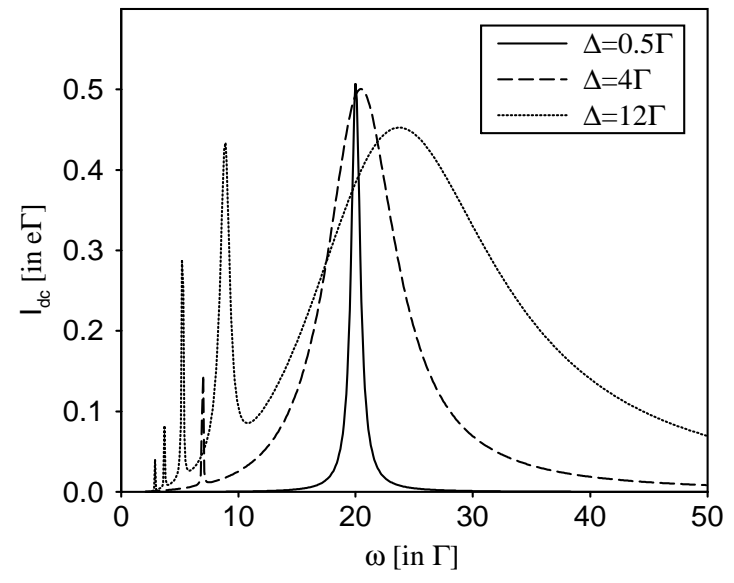

FIG. 2. Current response of a double quantum-dot electron pump versus frequency $\omega$ for a time-dependent barrier separating the dots. $\Delta_{0}=0 \Gamma \Delta_{D}=0$. 

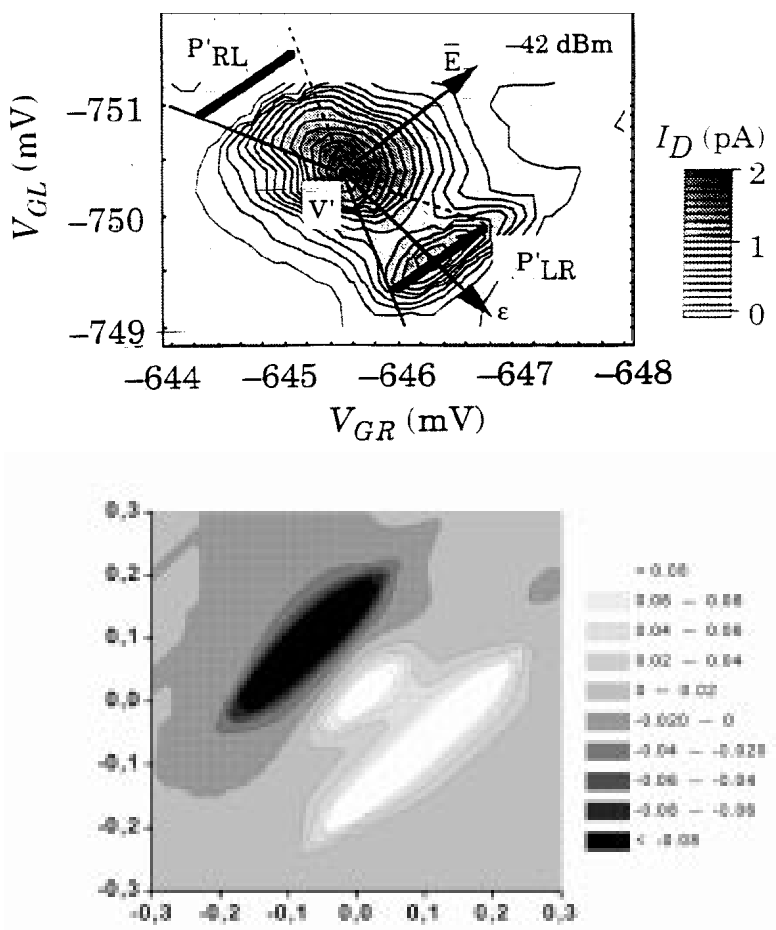

FIG. 3. Experimental results of Fujisawa and Tarucha ${ }^{5}$ showing the current through a double quantum dot with a time-dependent interdot barrier (upper figure) as a function of the gates voltages applied to the left and right dot. Comparison to our results (lower figure) shows qualitative agreement. In the lower figure $\Gamma$ the gate voltages are given in units of $e / C_{g}$ Tthe current in units of $e \Gamma . \omega=25 \Gamma \Gamma \Delta_{0}=2 \Gamma \Gamma \Delta=3 \Gamma \Gamma \Delta_{D}=0 \Gamma \mu_{L}=2.5 \Gamma \Gamma \mu_{R}=-2.5 \Gamma \Gamma T=3 \Gamma$. 